Revista Multidisciplinar do Nordeste Mineiro, v.2 2019/02

ISSN 2178-6925

\title{
ASSISTÊNCIA DE ENFERMAGEM EM SITUAÇÕES DE DESASTRES AMBIENTAIS
}

\section{NURSING CARE IN ENVIRONMENTAL DISASTER SITUATIONS}

Letícia Gomes Pereira; Acadêmica do curso de Enfermagem da Faculdade Presidente Antônio Carlos de Teófilo Otoni - MG. Email: lehgomes@outlook.com

Tarik Portugal Salman de Oliveira Acadêmica do curso de Enfermagem da Faculdade Presidente Antônio Carlos de Teófilo Otoni - MGLehgomes@outlook.com

\begin{abstract}
Aliny Gonçalves Batista
Enfermeira pela Universidade Presidente Antônio Carlos (UNIPAC), Mestre em Ciências Biológicas pela Universidade Vale do Rio Doce, Especialista em Gestão de Saúde Pública e Epidemiologia; Especialista em Gestão Microrregional em Saúde; Especialista em Regulação em Saúde no SUS; Docente da Faculdade Presidente Antônio Carlos de Teófilo Otoni/MG. E-mail: enfalinyperoba@hotmail.com
\end{abstract}

\section{Resumo}

Esse artigo caracteriza-se como uma revisão narrativa de resultados e experiências publicadas na literatura científica por meio da base de dados Scielo sobre a descrição da atuação do profissional de enfermagem no atendimento às vítimas de desastres ambientais e ou naturais. Desastres naturais são caracterizados como consequências de alterações na natureza influenciadas ou não pelo ser humano e afetam diversas regiões do mundo. Além do impacto ambiental direto, geram também importante efeito político, econômico-social e cultural modificando a dinâmica da sociedade afetada. O cuidado de saúde, nestes casos, envolve de forma significativa a atuação dos profissionais de enfermagem através da promoção de atendimento, acolhimento e a garantia de informações que esclareçam os impactados. A fase de transição é considerada fundamental para dar início a fase pós-desastre. Diante disso identificou-se a necessidade de aprimoramentos nos atendimentos de emergências de saúde pública no Brasil e em sua continuidade. A

\footnotetext{
*Aluna do $9^{\circ}$ período do Curso de Enfermagem da Universidade Presidente Antônio Carlos UNIPAC Teófilo Otoni - MG - e-mail: lehgomes@outlook.com

**Aluno do $9^{\circ}$ período do Curso de Enfermagem da Universidade Presidente Antônio Carlos UNIPAC Teófilo Otoni - MG - e-mail: Tarikportuga@ hotmail.com

***Enfermeira. orientadora. Mestre em Ciências Biológicas - Imunopatologia de doenças Infecciosas e parasitárias, Especialista em Gestão Saúde Pública e Epidemiologia, Especialista em Gestão Microrregional em Saúde, Especialista em Regulação em Saúde no SUS, Coordenadora Vigilância em Saúde Ouro Verde de Minas, Professora na Universidade Presidente Antônio Carlos UNIPAC - MG - e-mail: enfaliny.peroba@ hotmail.com
} 
diminuição dos impactos causados pelo desastre com os atendimentos de emergência possui ligação direta com a capacitação dos profissionais de enfermagem com o objetivo de prestar atendimentos de qualidade. A atuação da enfermagem é de suma importância em situações de desastres ambientais e ou naturais. É de fundamental importância a atuação do enfermeiro no atendimento às situações vivenciadas por cada familiar durante ou após o acontecimento do desastre sendo o mesmo o responsável pelo planejamento e treinamento da equipe predeterminando as mudanças em relação a promoção de saúde em setores hospitalares ou de Atenção Primária.

Palavras-Chave: Desastres ambientais. Enfermagem Familiar. Planejamento em Desastres. Assistência de enfermagem.

\begin{abstract}
This article is characterized as a narrative review of results and experiences published in the scientific literature through the Scielo database on the description of nursing professionals' performance in the care of victims of environmental and or natural disasters. Natural disasters are characterized as consequences of changes in nature influenced or not by humans and affect various regions of the world. In addition to the direct environmental impact, they also generate important political, economic, social and cultural effects by changing the dynamics of the affected society. Health care, in these cases, significantly involves the performance of nursing professionals through the promotion of care, reception and the guarantee of information that clarifies the impacted ones. The transition phase is considered key to initiating the post-disaster phase. Given this, we identified the need for improvements in public health emergency care in Brazil and its continuity. The reduction of the impacts caused by the disaster with emergency care is directly linked to the training of nursing professionals to provide quality care. Nursing performance is of paramount importance in situations of environmental and or natural disasters. It is fundamentally important for nurses to act in response to situations experienced by each family member during or after the disaster, being responsible for the planning and training of the team, determining the changes in health promotion in the hospital or primary care sectors.
\end{abstract}

Keywords: Environmental Disasters. Family Nursing. Disaster Planning. Nursing care.

\title{
1 Introdução
}

O impacto de desastres naturais e ou ambientais possui força capaz de traumatizar as vidas das vítimas, famílias e comunidades. No Brasil, apesar do crescimento do número e intensidade dos desastres, não existe aceitação formal em nível nacional da necessidade de integrar conteúdo sobre desastres nas Diretrizes Curriculares de Enfermagem ou desenvolver um programa de educação continuada padronizado. (1) 
Enfermeiros são profissionais que precisam apresentar competências com a finalidade de prestar assistência em situações estáveis, tendo importante atuação durante atendimentos de emergência, trabalhando nos cenários pré-hospitalares, hospitalares e pós-hospitalares, contribuindo para salvar vidas e promover a saúde em condições tão difíceis. (2)

A enfermagem é uma das profissões mais envolvidas no atendimento aos afetados em desastres naturais, com foco nas ações de urgência e emergência para respostas ágeis. A Organização Mundial da Saúde (OMS) tem divulgado informações sobre as ações da enfermagem no setor de saúde pública, com ênfase na reestruturação dos serviços ou programas de saúde e na recuperação do estado de saúde de populações em situações pós-desastres. Iniciando o direcionamento aos enfermeiros na Atenção Primária de Saúde (APS), e especificamente na política brasileira da Estratégia de Saúde da Família (ESF), pois educação e treinamento constituem o fundamento da preparação para os desastres. $(1,3,4)$

Sendo assim a abordagem metodológica deste trabalho consiste em uma pesquisa qualitativa de descrição bibliográfica sobre o tema por meio de leituras de livros, periódicos e pesquisas em sites da internet com a finalidade de compreender e descrever as atividades de organização, preparação, capacitação, promoção e prevenção gerenciadas pelo coordenador de enfermagem em situações de emergência em Saúde Pública em setores intra-hospitalares e de Atenção Primária visando a humanização nos atendimentos, prevenção de sequelas invisíveis e cuidados para evitar a propagação de doenças pós desastres.

\section{Conceitos básicos sobre desastres ambientais}

Desastres ambientais são definidos a princípio como fenômenos naturais, que independem ou não de interferências do ser humano sobre o meio ambiente, são conceituados como eventos de risco, sendo seus danos determinados de acordo com a intensidade de suas consequências, desenvolvimento, planejamento e infraestrutura da região afetada. (1)

Segundo Bergeron, Bizjak, Krause, Baudour (5) são consideradas situações de desastres aquelas em que é impossível atender a demanda de acidentes com múltiplas vítimas (AMV) ativando assim o sistema de Gerenciamento de Desastre. 
Nunes (6) classifica esses eventos como ameaça ao desenvolvimento real, tendo em vista que as perdas infligidas por eles podem comprometer esforços de décadas, tendo dois processos sociais contemporâneos que podem alterar rapidamente o ambiente natural da urbanização e a globalização.

No ponto de vista da saúde pública, os desastres são definidos de acordo com as sequelas deixadas aos seres humanos e serviços de saúde. Nos termos do inciso II do artigo $2^{\circ}$ do Decreto 7.257, de 4 de agosto de 2010, desastre é definido como o "resultado de eventos adversos, naturais ou provocados pelo homem sobre um ecossistema vulnerável, causando danos humanos, materiais ou ambientais e consequentes prejuízos econômicos e sociais" (7)

Toda a humanidade está sujeita a sofrer com desastres naturais, e estes serão considerados situações de emergência ou estado de calamidade pública somente após análises de fatores principais como intensidade dos danos (humanos, materiais e ambientais) e estudo dos prejuízos sociais e econômicos em comparação aos recursos antes disponíveis na região afetada. (8)

Por fim desastres ambientais são ocorrências violentas não previsíveis que rompem o cotidiano padrão de cidadãos da região afetada, capazes de direta ou indiretamente causar danos ao meio ambiente e a saúde humana ocasionando traumas em toda a população envolvida.

\subsection{Classificação Médica da Intensidade dos Desastres}

Em função do número de vítimas, da gravidade das lesões corporais e da disponibilidade local dos recursos de saúde, os desastres são classificados em:

Acidentes ou Desastres de Pequeno Porte: Quando a unidade de emergência do hospital responsável pelo apoio local à área onde ocorreu o desastre tem condições de atender às emergências médicas e cirúrgicas consequentes, sem alterar sua rotina diária. (9)

Desastres de Médio Porte: Quando a gravidade das lesões corporais e o número de vítimas obrigam o hospital responsável pelo apoio local desencadeie o plano de mobilização e expansão da capacidade de atendimento emergencial. (9)

Desastres de Grande Porte: Quando exigem uma resposta sistêmica, envolvendo mais de uma instituição hospitalar e exigindo a mobilização dos recursos de saúde na região de saúde à qual pertence a área afetada. (9) 
Desastres de Muito Grande Porte: Quando é necessário que sejam agregados reforços sanitários de outras regiões de saúde, do Estado, do país e, em situações extremas, recursos internacionais. Nesses casos, pode-se estabelecer um sistema de evacuação aero médica para aliviar os hospitais da região afetada. (9)

\section{Os desastres ambientais no Brasil}

Os desastres ambientais de consequências naturais e de grande extensão (tsunamis, terremos) raramente ocorrem no Brasil, geralmente eles são motivados pela ação do homem. De acordo com o IBGE as secas são o tipo de desastre que mais prejudicam grande parte dos municípios brasileiros (48,6\%), em segundo lugar está o alagamento (31\%) e em terceiro enchentes ou enxurradas (27\%). (10)

De acordo com alguns estudos no período entre 2004 a 2019 todos os estados brasileiros, com exceção do Distrito Federal, tiveram processos de situação de emergência ou estado de calamidade pública reconhecidos pelo Ministério da Integração Nacional sendo os municípios pequenos, com até 50.000 habitantes, os mais afetados por esses fenômenos. (11)

Eventos de desastres ambientais no Brasil possuem ocorrências recentes com intervalo de frequência cada vez menor:

2004: Nos dias 27 e 28 de março o ciclone Catarina, que afetou o norte do Rio Grande do Sul e o sul de Santa Catarina, deixou danos nocivos à saúde humana foram no total 27.560 desalojados, 2.589 desabrigados, 3.016 deslocados, 518 feridos e 1 óbito. (11)

2007: No dia 10 de janeiro a barragem de rejeitos de lavra de bauxita situada na zona rural do Município de Miraí em Minas Gerais, se rompeu, causando sérios riscos ambientais ao município, houve inundação de aproximadamente 400 casas e comércios, cerca de 2 mil pessoas desalojadas e desabrigadas, não houve nenhum caso de óbito. (12)

2008: Em novembro o Vale do Itajaí foi assolado por índices altíssimos de chuva, precipitações, inundações, enxurradas e escorregamentos. $O$ evento atingiu a cidade de Blumenau (SC). Foram atingidos mais de 1,5 milhão de catarinenses, 135 mortos, cerca de 80.000 desabrigados ou desalojados. No total, 14 municípios decretaram estado de calamidade pública, e 63 situações de emergência. O sistema 
de saúde precisou suspender o atendimento de rotina para focar na emergência. $(13,14)$

Um aumento significativo de casos de acidentes vascular-cerebrais foi observado após as enchentes, o que pode ser consequência, tanto de situações de estresse e transtornos pós-traumáticos, quanto da perda de vínculo com os sistemas de atenção básica de saúde. (15)

2010: Enchentes dos rios Una e Jaboatão devido às chuvas intensas em Pernambuco e Alagoas afetaram 28 municípios, dos quais quatro decretaram situação de emergência e 15 municípios encontravam-se em estado de calamidade pública. Foram afetadas 181.018 pessoas, das quais 26 foram a óbito, 69 desaparecidos, 26.618 desabrigados, 47.847 desalojados. Diante dessa situação o Ministério da Saúde do Brasil solicitou ao Grupo Hospitalar Conceição, profissionais da saúde para operarem como voluntários sendo que a unidade hospitalar em situações de desastres anteriores já havia providenciado funcionários para atendimentos. Nos meses de junho e julho, cerca de 40 profissionais da área da saúde se inscreveram, entre auxiliares e técnicos de enfermagem, enfermeiros e médicos para comporem equipes de atuação direta junto à população das cidades acometidas pelas enchentes. (16)

2011: No dia 11 de janeiro na região Serrana do Rio de Janeiro houve o maior desastre climático do Brasil sendo registrado na época pela a ONU como o $8^{\circ}$ pior deslizamento da história mundial. Foram registrados 918 óbitos, 8.795 desabrigados e 22.604 desalojados, resultando no impacto direto sobre a vida de mais de 32 mil habitantes, com o total de sete municípios decretando estado de calamidade pública. As ocorrências de traumas notificadas no serviço municipal de emergência triplicaram na semana posterior ao desastre, mas não houve aumento da demanda por serviços de reabilitação. $(17,18)$

2015: No dia 05 de novembro houve o rompimento da barragem de Fundão da Samarco em Mariana (MG) deixando 17 mortos, 2 desaparecidos, 256 feridos, 380 enfermos, 644 desabrigados, e cerca de 1,2 milhões de pessoas sem água. Um ano após a tragédia, a contaminação da água do rio Doce utilizada para consumo humano ainda apresenta risco. Após o desastre, a lama seca gerou exposição a poeira rica em ferro e sílica que desencadeia processos alérgicos, principalmente cutâneos e respiratórios, como ocorrido em Barra Longa, município vizinho de 
Mariana, onde foi verificado um grande número de ocorrências de infecções respiratórias, cutâneas e conjuntivites nesta população. (19, 20, 21)

2019: No dia 25 de janeiro a Barragem I da mina de Feijão em Brumadinho (MG) rompeu-se. Os dados da Defesa Civil confirmam que 727 pessoas foram expostas a risco de vida e ficaram em situação de desaparecidas no dia do rompimento da barragem. A força da onda da lama resultou na morte de 134 pessoas e outras 199 seguem desaparecidas. $(21,22)$

O primeiro desastre natural registrado na história de Teófilo Otoni (MG) ocorreu em dezembro de 1992, devido forte chuva que ocasionou uma grande enchente. Em 04 de fevereiro de 2002, ocorre o segundo desastre ambiental na cidade também ocasionado por forte chuva que gerou inundações agravadas pelo transbordamento dos dois rios que cortam o município, sendo: Rio Santo Antônio e Rio Todos os Santos. A Coordenadoria de Defesa Civil na época contabilizou 70 desabamentos de casas, vários deslizamentos, cerca de 8.000 pessoas ficaram desabrigadas e 12 foram a óbito, essas pessoas residiam em sua maioria em áreas de risco na encosta da cidade.A Defesa Civil afirmou ter enviado medicamentos para evitar doenças que podem se propagar com a inundação. $(23,24)$

\section{Desastres ambientais e seus potenciais impactos na saúde pública}

A Saúde é um bem imaterial garantido pela Constituição Federal de 1988, em seu artigo 196, in verbis: Art. 196. "A saúde é direito de todos e dever do Estado, garantido mediante políticas sociais e econômicas que visem à redução do risco de doença e de outros agravos e ao acesso universal e igualitário às ações e serviços para sua promoção, proteção e recuperação". (25)

As consequências dos desastres podem afetar diretamente a saúde e o bemestar da comunidade, resultando em enfermidades e óbitos. Os desastres ambientais prejudicam o meio ambiente, em vários aspectos como: os serviços de saneamento ambiental, o abastecimento de água, a distribuição de alimentos, alterações de vetores e hospedeiros. A infraestrutura local também é afetada, prejudicando a economia, a sociedade local, sendo que os próprios serviços de saúde podem ter sua assistência comprometida, enfatizando então a importância que os serviços de saúde essenciais continuem em andamento para garantir a 
assistência integral a população, principalmente logo após a ocorrência de um desastre. $(1,26,27)$

A suspensão do atendimento dos serviços de saúde por destruição em desastre é lesiva, sendo definido como ação prioritária que todas as unidades de saúde tenham uma estrutura física, compacta aos impactos dos fenômenos naturais e ou ambientais.(28). Há a necessidade de que os métodos de prevenção de danos dos equipamentos, serviços fundamentais (água, eletricidade, gases medicinais, coleta dos resíduos de saúde, saneamento, telecomunicações, acesso, medicamentos etc.) permaneçam operando e que a equipe de saúde continue exercendo atenção médica em situações que demandem cuidados que os indivíduos mais necessitem. De acordo com o tipo de fenômeno que provoca o desastre os danos atingem os pacientes, visitantes e profissionais de saúde que se encontram naquela unidade atingida. (29)

Os impactos resultantes de curto a longo prazo, resultam do tipo de evento e fragilidade socioambiental da região atingida. O período para surgimento dos acontecimentos resultantes de um desastre se diversifica entre algumas horas a anos. A curto prazo que é determinado por horas e dias os serviços realizados são de registros de feridos leves, graves, mortalidade e resultados das atividades de resgate e urgência. $(1,30,31)$

Em situações de desastres de origem ambiental, como as inundações, potencializam a ocorrência de doenças, sobretudo as transmitidas por água, alimentos, vetores, reservatórios e animais peçonhentos. (1)

Já a longo prazo que é determinado por dias a semanas os serviços realizados são de constatação e notificação de doenças transmissíveis, como: leptospirose, doenças diarreicas e complicação de doenças não transmissíveis como hipertensão em doentes crônicos. Sendo relevante dar início as ações de vigilância, prevenção e recuperação dos serviços à assistência à saúde para controle de doenças. Os problemas de saúde que atingem os sobreviventes a longo prazo após a ocorrência do desastre são relevantes como: depressão, problemas psicológicos, abusos de substancias químicas e outros tipos de consequências sendo o cuidado direcionado à Atenção Básica, afetando mulheres, crianças, jovens e idosos. (30)

No decorrer de meses e anos, os impactos na saúde pública ocasionados por desastres ambientais possuem relação com doenças não transmissíveis, em 
particular os transtornos psicossociais e comportamentais, doenças cardiovasculares, desnutrição e ao aumento de doenças crônicas (1)

Conforme dados da SDNC 2003-2012 são considerados efeitos mais comuns sobre a saúde humana decorrentes de desastres naturais: óbitos, lesões, traumatismos, intoxicação, envenenamento, transtornos psicossociais e comportamentais e hipertensão em desastres como terremoto ou vulcão. Já em desastres do tipo tempestade, ciclone ou vendaval há acréscimo de efeitos como afogamento e choque elétrico. Em situações de deslizamentos, alagamento ou inundação brusca e gradual: Todos os efeitos citados juntamente com a ocorrência de doenças como: leptospirose, doenças transmitidas por vetores, reservatórios e hospedeiros, desnutrição, diarréia e infecções intestinais, infecções cutâneas e hepatite A. Em situações de desastres com fenômenos como seca, frio ou incêndio florestal: infecções respiratórias agudas e crônicas, alergia e raquitismo. (11)

É significativo expor e esclarecer os acontecimentos, para estimular e incentivar profissionais, e acadêmicos da enfermagem a disporem da oportunidade de exercerem a assistência de enfermagem como voluntários ou profissionais em tarefas para o bem-estar dos cidadãos possibilitando qualidade da saúde humana aos afetados pelos desastres, apresentando a atuação de uma equipe de saúde.

\section{Atuação do enfermeiro}

O enfermeiro possui dever necessário e indispensável no plano de desastre sendo ele ponderado pela organização, preparação e capacitação da equipe, um aspecto essencial no atendimento às múltiplas vítimas. O profissional da enfermagem deve programar todas as ações da equipe de enfermagem, optando pela assistência à pessoa afetada de forma integral. (31)

De acordo com o tipo de desastre e sua característica é determinado o padrão de traumatismo, como nos desastres ferroviários que ocasionam mutilações que geram monoplegia ou monoparesia dos membros ou em desastres aéreos em que a maioria das vítimas sofrem com queimaduras e poli traumatismos, tornando-se essencial que o profissional de enfermagem tenha uma visão global sobre o tipo e os possíveis traumas decorrentes de cada desastre para prestar uma melhor assistência e provisão de recursos materiais. (31) 
A ação fundamental em cuidados frente a um incidente com múltiplas vítimas (IMV) é proporcionar o bem máximo para o número máximo de pessoas. Disponibilizando o melhor cuidado médico para o maior número possível de vítimas, o que envolve o momento certo, o tempo adequado e a utilização mínima de recursos, isto é, uma atuação profissional eficiente e precisa. $(2,32)$

Em situação de ocorrência de um desastre ambiental o papel do profissional de enfermagem nos cuidados críticos é indispensável. O cuidado minucioso nesta situação está ligado a intensidade do impacto causado pelo desastre sobre as estruturas das instituições e o número de profissionais disponíveis para prestar o atendimento às vítimas (32). A atuação do enfermeiro depende de muita disciplina, entendimento da situação e comprometimento durante a assistência e atendimento as vítimas, nesse sentido o enfermeiro deve estar ciente de suas funções de gerenciamento e didática, particularmente em situações inusitadas como em desastres com grande número de vítimas que chegam a unidade hospitalar, para que junto com a equipe multidisciplinar consigam amplificar a assistência de modo mais apropriado. (33)

A Resolução COFEN nº 293/2004, fixa e estabelece parâmetros para o dimensionamento do Quadro de Profissionais de Enfermagem nas Instituições de Saúde, sendo que mais da metade dos profissionais destas instituições são formados pelos profissionais de enfermagem, cabendo ao enfermeiro proporcionar condições para que ocorra o envolvimento da equipe de enfermagem e assim executem sua função da melhor maneira possível, contribuindo para a qualidade da assistência prestada. (34)

Em qualquer ocasião a prestação de serviços de saúde precisa da sincronia com equipe multidisciplinar, tendo o enfermeiro papel fundamental na assistência, por isso é necessário desenvolver conhecimentos, habilidades e atitudes, de modo a garantir o atendimento qualificado e integral. Nos atendimentos a situações de calamidade pública, a enfermagem desempenha um papel central. (35)

A ajuda humanitária é considerada como uma forma de cooperação sendo exercida de várias formasno atendimento às necessidades básicas ou de urgência, como: alimentação, saúde e reconstrução das infraestruturas devido a algum desastre. Essa ajuda se diferencia na urgência e nas necessidades de cada país 
podendo ser material, econômica ou profissional para trabalho voluntário diretamente na região afetada. (18)

De acordo com isso podemos identificar a necessidade de o enfermeiro estar preparado e disposto para colocar o seu saber a disposição do seu semelhante, pois ele está intensamente envolvido com a vida e com o ser humano. Sendo a enfermagem uma a ciência do cuidado integral.

\subsection{Formação Acadêmica: O Alicerce Fundamental}

A proteção ás vítimas de desastres ambientais ou naturais minimiza as consequências catastróficas desses eventos, aumentando os índices de sobrevida das vítimas. Daí, a formação do acadêmico da área da saúde se torna fundamental pois é considerada como ferramenta de treinamento e aperfeiçoamento. (3)

O Brasil possui grande avanço no setor da Saúde Pública, mas quando se trata de situações de desastres deixa a desejar. Identificando, portanto, a necessidade de a comunidade acadêmica aumentar a produção de conhecimentos e informações sobre a redução de desastres ou assistência aos desastres intensivos mais ocorrentes no Brasil, como inundações bruscas e deslizamentos, mas também dos desastres extensivos, particularmente a seca, investimentos em estudos sobre a atuação do setor de saúde nas etapas de redução do risco, compreensão dos impactos e efeitos sobre a saúde da população exposta aos desastres. (3)

Nos últimos anos, a necessidade de informações sobre o tema desastres ganhou tanta importância no cenário internacional, que a Biblioteca Virtual em Saúde (BVS) desenvolveu o portal "Preparação e resposta a desastres", o qual inclui, entre outras, a base "Desastres". A intenção é que o portal possa tornar ainda mais acessível a produção científica, material técnico, guias práticos e planos de preparação, bem como informações ao público e facilitar o estabelecimento de redes sociais, ampliando a proposta e as possibilidades da base "Desastres". $(3,33)$

Em atendimento a Emergências de Saúde Pública, o profissional da enfermagem atua em todos os níveis de atenção, pois são estes profissionais que iniciam o processo da assistência (36). A resposta a Emergência de Saúde Pública necessita que a equipe de enfermagem tenha um preparo específico. (33)

Muitos profissionais não se sentem preparados para prestar atendimentos em situações de desastres por deficiência de conhecimento em fatores como: 
habilidades, assistência aos sobreviventes e suporte para a saúde mental dando ênfase a necessidade da educação e treinamento científico das universidades promovendo oportunidades educacionais e sensibilizando alunos e profissionais para os planos institucionais de gestão de desastres, desenvolver programas de capacitação para os profissionais de saúde e aqueles envolvidos no trabalho humanitário criando assim recursos que podem ser utilizados como embasamento de preparação e resposta através de parcerias acadêmicas e a saúde pública. (3)

Também é necessário que a didática utilizada seja adequada para que os acadêmicos possam desempenhar com êxito as suas funções em desastres, sendo necessário instituir uma disciplina como exigência profissional. Assim os enfermeiros concluiriam a graduação capazes de demonstrar suas competências, fornecendo assim um caminho para preparar adequadamente outros profissionais para seus futuros papéis na gestão de desastres. (3)

Portanto entende-se que os acadêmicos da área da saúde obtendo uma didática na assistência a desastres podem oferecer ajuda humanitária pois terão percepção das competências que precisam ser exercidas oferecendo um cuidado seguro e eficiente.

\section{Atendimento hospitalar pós desastre}

A chegada das vítimas consideradas como mais graves aos serviços de emergência ocorre em um curto espaço de tempo, geram grande estresse aos profissionais de saúde que recebem uma onda de pacientes e leva a sobrecarga dos funcionários e o hospital precisa estar rapidamente preparado para dar lugar às vítimas. Apoio emocional e acolhimento dos pacientes e familiares também são preocupações destes profissionais. (37)

No contexto hospitalar, o planejamento e o aumento da capacidade de atendimento em situação de desastre prevê a atuação da equipe de enfermagem em todas as etapas, desde a triagem das vítimas, até a transferência do centro cirúrgico para a unidade de tratamento intensivo (UTI) $(6,18)$.

A Sistematização da Assistência de Enfermagem (SAE) é uma ferramenta importante na assistência ao indivíduo. É exclusiva do enfermeiro e tem como objetivo o desenvolvimento e organização do trabalho da equipe de enfermagem, fortalecendo a profissão (38). 
A triagem é uma das principais fases nos atendimentos a vítima, realizada corretamente determina o índice da morbidade e mortalidade (39). Uma instituição hospitalar está inserida no Ciclo de Desastres, como um órgão de resposta, com profissionais capacitados para atendimento ás vítimas de desastres a resposta ocorrerá de forma efetiva e satisfatória, reduzindo assim as consequências dos desastres, onde o objetivo é garantir a integridade da vida humana. $(3,40)$

Esses pacientes estão expostos a comorbidades individuais que possuem grande influência no prognóstico, devido as consequências de os desastres acarretarem traumas. Por isso, é importante aplicar os protocolos de triagem e reconhecer a classificação feita pelo atendimento pré-hospitalar, por seleção por prioridade quanto ao quadro clínico e constante monitorização evitando assim uma iatrogenia e também para estabelecer uma ação interna efetiva a fim de reduzir a morbi-mortalidade das lesões. $(10,40)$

A qualidade do atendimento ao paciente na situação de emergência depende da qualificação do profissional enfermeiro e de toda a equipe, da calma e do bom senso para evitar condutas tumultuadas, estressantes, prevenir intercorrências e evitar maiores complicações. (32)

É necessário recordar que o enfermeiro responde pela conduta e desempenho do serviço dos seus colaboradores sendo realizados ou não com a presença do mesmo diante disso se torna indispensável enfatizar o bom treinamento da equipe com o objetivo de promover segurança aos mesmos e aos pacientes.

As unidades e instituições de saúde que dispõem de organização para atendimento de múltiplas vítimas em desastres habitualmente possuem protocolos de normas e procedimentos para atendimento específico para tais situações como, por exemplo, os checklists que são elaborados pelo enfermeiro coordenador para agilizar o atendimento e orientar membros da equipe que não possuem prática diante destes eventos. $(10,32)$

Em casos de carência de recursos materiais e fonte de energia para procedimentos de emergência é necessária a capacitação especializada para a realização destes procedimentos, demostrando assim, eficiência no atendimento. (32)

Pode-se dizer que a atuação efetiva do enfermeiro coordenador e toda a equipe de enfermagem é como um conceito para o sucesso dos atendimentos com o 
objetivo de prestar assistência de qualidade e garantir a segurança dos clientes. $O$ cuidado é exercido com a atuação permanente da equipe de enfermagem seja o técnico de enfermagem ou o enfermeiro prestando assistência ou gerenciando. (32)

A equipe multidisciplinar prestando atendimento de qualidade evita condutas desordenadas e de esgotamento, portanto torna-se necessário divulgar condutas e atitudes do enfermeiro no gerenciamento e atendimento a vítimas de desastres.

\section{Papel da atenção básica e as mudanças de rotina no atendimento as famílias no pós desastre}

A Atenção Básica $(A B)$ é caracterizada com a associação de ações individuais e coletivas na área da saúde, que abrangem a promoção, prevenção de agravos, o diagnóstico, o tratamento e a reabilitação com o objetivo de desenvolver uma atenção integral que impacte positivamente na situação de saúde das coletividades (41). A implantação da $A B$ somente é realizada com bons resultados caso haja trabalho em equipe o que não é considerada uma ação de fácil acesso, mas é fundamental para atingir os objetivos de qualidade em saúde. (42)

As ações de planejamento da assistência são determinadas de acordo com as áreas afetadas, os níveis de vulnerabilidade destas, o tipo de desastre, condições sociais, econômicas e de saúde dando enfoque nos principais problemas (43). No Brasil, as famílias sobreviventes aos desastres inicialmente são encaminhadas a abrigos públicos até a fase de reconstrução do desastre para estabelecer um ambiente seguro e saudável (44). O tempo de morada nesses abrigos pode estender-se de semanas a meses, neste período são elaborados programas de mitigação e reforço pois no Brasil a fase de preparação de enfrentamento a desastres ambientais não é sólida. $(9,45)$

O período de transições familiares pós desastre principalmente nos desastres inesperados, produz grande estresse ocasionando efeitos nos status da saúde. Em devida circunstância os enfermeiros podem ser os únicos com contato direto em uma relação de ajuda as vítimas fornecendo informações, apoio e disponibilizando recursos. A promoção da saúde é uma função primordial da enfermagem familiar ou AB (45).

$A$ produção da $A B$ é incorporada com base na sociedade e rotinas da comunidade, que são os meios mais importantes utilizados pelos enfermeiros para 
verificar comportamentos e conhecimentos com os integrantes, identificar metas familiares e criar planos para alcançar o bem-estar ou iniciar um processo para a implementação de práticas saudáveis. Esses meios também fornecem estratégias de cuidados necessários na doença aguda ou crônica, para a proteção e a promoção da saúde, especialmente das crianças $(49,50)$.

Assim, a equipe da $A B$ seguindo orientações do enfermeiro da unidade deve promover serviços de prevenção, acolhimento, cuidado e tratamento com o objetivo de prestar um cuidado integral, incluindo em situações de desastres, quando a demanda pelos serviços de saúde pode aumentar. Buscar a organização dos serviços para a continuidade do seu funcionamento possui grande influência na redução de risco à saúde da comunidade afetada. (31)

As principais ações do enfermeiro no setor de $A B$ em situações de desastres são:avaliar danos e necessidades, retomar os serviços de rotina, integrar serviços com a vigilância em saúde, identificar e recadastrar as famílias atingidas, atender as vítimas nos abrigos, desenvolver ações de busca ativa de casos agudos e crônicos, notificar eacompanhar casos suspeitos e confirmados de agravo no Sistema de Informação de Agravos de Notificação (SINAN), manter atualizado o (ESUS) e o Sistema de Vigilância Alimentar Nutricional (SISVAN) ou outros sistemas utilizados na $A B$, realizar avaliação da situação da saúde em conjunto com as outras equipes e reorganizar o atendimento da Rede Básica de Saúde. (31)

Vigilância da violência nos abrigos (física e/ou sexual), detecção de surtos e epidemias, monitoramento de doenças e agravos, notificação ao Centro de Informações Estratégicas de Vigilância em Saúde (CIEVS), investigação e ações de controle. (31)

Monitoramento e notificação de morbimortalidade nos Sistemas de Informação (SINAN, SIM), monitorar dados de outros sistemas de informação e acompanhamento do perfil epidemiológico. (31)

As principais ações em situações de inundações são voltas a vigilância e controle de doenças transmissíveis, monitoramento de doenças diarreicas agudas, leptospirose, hepatiteA, doenças de transmissão respiratória, tétano acidental, doenças transmitidas por vetores, transtornos psicossociais, doenças crônicas descompensadas, acidentes com animais, desnutrição, acidentes (afogamento, choque elétrico, quedas, soterramento etc.). (31) 
Imunização: Caso seja necessário remanejar os imunobiológicos, avaliação de perda de insumos, manutenção da rede de frio (infraestrutura e equipamentos), garantir e definir intensificação de imunização e identificar os casos especiais. (31)

Vigilância da qualidade da água para consumo humano: Avaliar os danos nos Sistemas de Abastecimento de Água (SAA) e Soluções Alternativas Coletivas e Individuais (SAC e SAI), avaliar e assegurar a quantidade e qualidade da água nos abrigos temporários, identificar em articulação com o responsável pelo abastecimento de água para consumo humano, outras fontes seguras de abastecimento. Controle higiênico-sanitário de abrigos: avaliar as instalações físicas e a capacidade instalada dos abrigos. (31)

\subsection{Serviços e Cuidados Direcionados a Reabilitação das Famílias Pós Desastres Ambientais}

Em situações de desastres ambientais é necessário intensificar as ações de monitoramento e vigilância em saúde com bom planejamento e associação com outros setores e com base em dados para a tomada de decisões (22).

Os serviços de cuidados voltados para a reabilitação após desastres naturais não são somente voltados a intervenções biomédicas, abrange também os aspectos sociais, econômicos, comportamentais e ambientais (1). $O$ objetivo dessas intervenções é promover um nível máximo de independência, funcionalidade e reintegração do sujeito à sociedade, é necessário orientar e estimular também a família, e não apenas o indivíduo. $(12,18)$

Pacientes com lesões graves podem voltar a ter uma vida produtiva, inclusive retornando ao mercado de trabalho, entretanto, é necessário passar por um processo de reabilitação complexo, que demanda cuidados de saúde especializados, trabalho em equipe e a atenção à saúde constante, de modo a evitar complicações imediatas e tardias. (18)

Em situações em que as vítimas sofreram lesões medulares são necessárias orientações do enfermeiro da ESF quanto aos cuidados relacionados à evacuação e micção, manutenção da integridade da pele, manejo da dor e adaptação às tecnologias. A reabilitação também pode se estender por toda a vida e tem relação 
com diferentes aspectos, inclusive nutricionais, psicológicos e culturais, podendo-se assim verificar a necessidade da AB. (18)

$O$ enfermeiro da ESF deve também comunicar aos sobreviventes da área junto a nutricionista do NASF sobre a necessidade de uma alimentação balanceada sendo importante para a manutenção da integridade da pele e recuperação das úlceras. Fatores psicológicos como depressão e sentimentos de raiva estão associados ao aumento da dor. (18)

A reabilitação necessita de um longo investimento e em grande parte dos países em desenvolvimento não há este cuidado após desastres ambientais, após o período de resgate, hospitalizações e buscas não há acompanhamento, sofrendo grande influência nas complicações clínicas. $(1,12,18)$

\section{Prevenção e redução de riscos}

O SUS dispõe de instrumentos que facilitam a articulação entre os diversos órgãos que estabelecem normas para a construção, gerenciamento e monitoramento dos estabelecimentos de saúde. Esses instrumentos facilitam o monitoramento dos estabelecimentos da rede de saúde pelas três esferas de gestão do SUS, permitindo a aplicação das gestões de risco corretiva e prospectiva. (15)

A partir de 2004 a Organização Mundial da Saúde (OMS) e a Organização Pan-Americana da Saúde (OPAS) vêm trabalhando no sentido de gerar uma consciência pública acerca do tema da segurança para os estabelecimentos de saúde com a proposta denominada "Hospitais seguros frente a desastres". (8)

Os países da América Latina e de todos os outros continentes adotaram essa proposta como uma política nacional de redução de riscos, com o objetivo de conseguir que novos estabelecimentos de saúde se construam com um nível de segurança que garanta sua capacidade de continuar funcionando, e podendo exercer um atendimento amplo nas situações de desastre, e que implantem medidas adequadas de mitigação para reforçar os estabelecimentos de saúde existentes, especialmente os que oferecem atenção primária. (8)

Para a OPS/OMS, um estabelecimento de saúde é seguro quando seus serviços permanecem acessíveis e funcionando em sua máxima capacidade instalada, e na mesma infraestrutura, logo após a ocorrência de um fenômeno destrutivo de origem natural. (23) 
O SUS ainda dispõe de um programa que se relaciona com o tema da rede de saúde: Programa Nacional de Vigilância em Saúde Ambiental dos Riscos Associados aos Desastres de Origem Natural (Vigidesastres). Dentre seus objetivos gerais consta "Reduzir os danos à infraestrutura sanitária e de saúde". (45)

As vidas humanas, os custos de reconstrução, as quedas do desenvolvimento confirmam que é necessário um plano de prevenção e redução de riscos de desastres ambientais com objetivo na diminuição de danos.

\section{Gestão de riscos de desastres ambientais no sus}

A gestão de risco de desastres é caracterizadapela agregação de tomada de decisões de organização e conhecimentos operacionais para implementar com o objetivo diminuir os impactos de desastres ambientais e tecnológicos consequentes. Gestão de risco de desastres no SUS contempla a gestão de projetos de investimento e a gestão ambiental. $(8,24)$

A gestão de risco de desastres subdivide-se em prospectiva, corretiva e reativa. A gestão prospectiva é uma prática que procura evitar erros do passado, estabelece uma relação imediata e direta com os processos de planejamento do desenvolvimento ao constituir o risco em um fator a considerar-se no estímulo e promoção de novos projetos, se desenvolve em função do risco ainda não existente e pode ser praticada por meio de novas iniciativas de investimento e desenvolvimento, estimuladas pelo governo, iniciativa privada, ONGs, associações de desenvolvimento, famílias ou indivíduos, é um componente integral da gestão do desenvolvimento. (28)

$\mathrm{Na}$ gestão corretiva sua prática tem como ponto de referência o risco já existente, produto de ações sociais diversas implantadas no passado, assentamentos humanos localizados em áreas. A intervenção corretiva pode ser de natureza conservadora, somente propondo ações sobre alguns dos fatores de risco identificados, sem pretensão de maiores transformações nos elementos sob risco. $\mathrm{Na}$ medida em que supera a intervenção conservadora e pontual e se constitui em uma intervenção transformadora, deve estar inserida no planejamento, para não promover ações avulsas. $(1,8,26,28)$. 
Em relação a um estabelecimento de saúde, a gestão reativa aplica-se quando as outras gestões (corretiva e prospectiva) não foram aplicadas. Nesse caso, resta formular um plano de resposta, com os recursos disponíveis. (28)

\section{Considerações finais}

A pesquisa apontou o quanto é essencial a atuação da enfermagem e sua equipe nos serviços de saúde e atendimentos relacionados frente a vítimas de desastres ambientais, com necessidade de estudos sobre estes atendimentos nos sistemas de saúde do Brasil na preparação dos profissionais de enfermagem para atuação em situações de desastres socioambientais, com objetivo de atender as demandas específicas de tais situações com rapidez, atendendo muitos pacientes no menor tempo e com a melhor qualidade possível.

A carência de planejamentos para tais situações e a não capacitação dos profissionais de saúde ocasionam complicações no momento do atendimento hospitalar que diante as ocorrências pode agravar o índice de mortalidade e sequela das vítimas. Os enfermeiros prestam atendimentos contínuos na fase de resposta, esta etapa requer cuidados de estabilização e homeostase.

Observou-se ainda a necessidade mais pesquisas com informações sobre atuação de enfermagem nos desastres a maioria dos pesquisadores mencionaram o fato da ausência de publicações referentes ao atendimento em desastres. Sugerese, que as instituições hospitalares e de Atenção Básica preparem e motivem suas equipes para atender pacientes vítimas de catástrofes.

Dessa forma, espera-se que este estudo possa servir de incentivo aos enfermeiros e agregar elementos que colaborem com a enfermagem na averiguação de informações sobre o reconhecimento da atuação da enfermagem em situações de desastres, ampliando esse conceito de atuação em desastre podemos identificar o enfermeiro como responsável pelo bem-estar das vítimas atendidas com o intuito de melhora a partir do início do atendimento, não atendendo somente aos direitos e deveres descritos nas normas impostas, mas também a segurança passada ao paciente para cessar a dor e garantir qualidade de vida.

\section{Referências}

1. Freitas CM, Silva DRX, Sena ARM, Silva EL, Sales LFB, Carvalho ML et al. Desastres naturais e saúde uma análise da situação do Brasil. Ciência \& Saúde 
Coletiva [Internet]. 2014 [acesso em 2019 jan 10]; 19(9): 3645-656. Disponível em: http://www.scielo.br/pdf/csc/v19n9/1413-8123-csc-19-09-3645.pdf

2. BuenoAA, Bernarde A. Percepção da equipe de enfermagem de um serviço de atendimento pré-hospitalar móvel sobre o gerenciamento de enfermagem. Texto Contexto Enferm. 2010 Jan-Mar; 19(1): 45-53. Disponível em: http://www.scielo.br/pdf/tce/v19n1/v19n1a05.pdf

3. Salvador PTCO, DantasRAN, DantasDV, Torres GV. A formação acadêmica de enfermagem e os incidentes com múltiplas vítimas: revisão integrativa. Rev Esc Enferm USP [Internet]. 2012 [acesso em 2019 jan 10]; 46(3):742-51. Disponível em: http://www.scielo.br/pdf/reeusp/v46n3/29.pdf

4. Ministério da Integração Nacional. Secretaria Nacional de defesa Civil. Universidade Federal de Santa Catarina. Centro Universitário de Estudos e Pesquisas sobre Desastres. Comunicação de riscos e de desastres. Florianópolis: CEPED; 2010. 183 p.93 http://www.crpsp.org.br/portal/comunicacao/diversos/mini_cd_oficinas/pdfs/Comunic acao-Riscos-Desastres.pdf

5. Bergeron JD, Bizjak G, Krause GW, Baudour CL. Acidentes de Grandes Proporções e Sistema de Gerenciamento de Desastres. In: Bergeron JD, Bizjak G, Krause GW, Baudour CL. Primeiros Socorros. $2^{2}$ ed. São Paulo: Ateneu Editora; 2007. p.525-540.

6. Nunes LH. Os desastres naturais - Condicionantes socioeconômicos e físicos. In: Nunes LH. Urbanização e Desastres Naturais. $1^{\underline{a}}$ ed. São Paulo: Oficina de Textos; 2015. p.13-24.

7. Brasil. Decreto 7.257, de 4 de agosto de 2010. II artigo. 2010. Dispões sobre o Sistema Nacional de Defesa Civil - SINDEC, sobre o reconhecimento de situação de emergência e estado de calamidade pública, sobre as transferências de recursos para ações de socorro, assistência às vítimas, restabelecimento de serviços essenciais e reconstrução nas áreas atingidas por desastre, e dá outras providências. [Internet]. [acesso em 2019 jan 10] Disponível em: http://www.planalto.gov.br/ccivil_03/_ato2007-2010/2010/decreto/d7257.htm

8. Fernandes GCM, Boehs AE. Rotinas de cuidado em relação à saúde de famílias em transição após um desastre natural. Rev. Latino-Am. Enfermagem. 2013; 21(4): 1-8. Disponível em: http://www.scielo.br/pdf/rlae/v21n4/pt_0104-1169-rlae-21-04-0982.pdf

9. Brasil. Ministério da integração nacional secretaria nacional de defesa civil. Castro ALC, Calheiros LB. Manual de medicina de desastres volume I. 2002. Brasília: Secretaria Nacional de Defesa Civil. 1996 104. [Internet]. [acesso em 2019 jan 10]. Disponível em: http://www.defesacivil.mg.gov.br/images/documentos/Defesa\%20Civil/manuais/medi cina-de-desastres.pdf

10. IBGE. Desastres naturais: $59,4 \%$ dos municípios não têm plano de gestão de riscos. [Internet]. [acesso em 2019 jan 10]. Disponível em: https://agenciadenoticias.ibge.gov.br/agencia- 
noticias/2012-agencia-de-noticias/noticias/21633-desastres-naturais-59-4-dosmunicipios-nao-tem-plano-de-gestao-de-riscos

11. Brasil. Ministério da Saúde. Organização Pan-Americana da Saúde. Desastres Naturais e Saúde no Brasil. Série Desenvolvimento Sustentável e Saúde 2. 2ª edição. 2015. p1-56. [Internet]. [acesso em 2019 jan 10]. Disponível em: https://www.paho.org/bra/images/stories/GCC/desastresesaudebrasil_2edicao.pdf

12. Ferreira DG, Santo RE. Responsabilidade civil ao dano ambiental no caso da mineradora rio pombo. [Internet]. [acesso em 2019 jan 10]. Disponível em: http://www.egov.ufsc.br/portal/sites/default/files/1775-1583-1-pb.pdf

13.Zenatti AAP, Sousa Soledad SYU. Relatos de um Desastre - Narrativas Jornalísticas da Tragédia de 2008 em Santa Catarina / Florianópolis: UFSC/CEPED, 2009. 132 p. [Internet]. [acesso em 2019 jan 10]. Disponível em:

http://www.ceped.ufsc.br/wp-content/uploads/2014/08/PR-745-Livro-Relatos-de-umDesastre-Miolo-101125.pdf

14. Avila MRR, Mattedi MA. Desastre e território: a produção da vulnerabilidade a desastres na cidade de Blumenau/SC. urbe. Revista Brasileira de Gestão Urbana (Brazilian Journal of Urban Management), 2017 maio/ago., 9 (2), p. 187-202. [Internet]. [acesso em 2019 jan 10]. Disponível em: http://www.scielo.br/pdf/urbe/v9n2/2175-3369-urbe-2175-3369009002AO03.pdf

15. Xavier DR, Barcellos C, Freitas CM. eventos climáticos extremos e consequências sobre a saúde: o desastre de 2008 em Santa Catarina segundo diferentes fontes de informação. Ambiente \& Sociedade. São Paulo v. XVII, n. 4; p. 273-294; out.-dez. 2014. [Internet]. [acesso em 2019 jan 10]. Disponível em: http://www.scielo.br/pdf/asoc/v17n4/a12v17n4.pdf

16. Márcio HS, Moema NF, Burg MRF, Gonçalves Bandeira, Andrea RWR. A equipe de saúde em missão humanitária no estado de Alagoas: Relato de Experiência [Internet]. [acesso em: 10 jan 2019]. Disponível em:

http://www.convencionsalud2018.sld.cu/index.php/connvencionsalud/2018/paper/vie wFile/2052/2251

17. Carvalho ML. Reabilitação física e recuperação da saúde no contexto dos desastres naturais: estudo de caso em Nova Friburgo. [Doutorado]. Rio de Janeiro: Escola Nacional de Saúde Pública Sergio Arouca; 2017. [Internet]. [acesso em 2019 jan 10]. Disponível em: https://www.arca.fiocruz.br/bitstream/icict/24104/2/mauren_lopes.pdf

18. Freitas CM, Carvalho ML, Ximenes EF, Arraes EF, Gomes JO. Vulnerabilidade socioambiental, redução de riscos de desastres e construção da resiliência - lições do terremoto no Haiti e das chuvas fortes na Região Serrana, Brasil. Ciência \& Saúde Coletiva. 2012; 17(6):1577-586. [Internet]. [acesso em: 10 jan 2019]. Disponível em: http://www.scielo.br/pdf/csc/v17n6/v17n6a21.pdf

19. Governo do estado de minas gerais secretaria de estado de desenvolvimento regional, política urbana e gestão metropolitana. Relatório: Avaliação dos efeitos e desdobramentos do rompimento da Barragem de Fundão em Mariana-MG. Decreto 
o 46.892/2015. Belo Horizonte Fevereiro de 2016. Disponível em: http://www.cidades.mg.gov.br/images/NOTICIAS/2016/relatorio_final.pdf

20. Lacaz FAC, Porto MFS, Pinheiro TMM. Tragédias brasileiras contemporâneas: 0 caso do rompimento da barragem de rejeitos de Fundão/Samarco. Rev Bras Saude Ocup 2017;42:e9. 1-12. Disponível em: http://www.scielo.br/pdf/rbso/v42/2317-6369rbso-42-e9.pdf

21. Romão A, Froes C, Barcellos C, Silva DX, Saldanha R, Gracie R, Pascoal V. Avaliação dos impactos sobre a saúde do desastre da mineração da Vale (Brumadinho, MG). Disponível em:

http://www.epsjv.fiocruz.br/sites/default/files/files/relat\%C3\%B3rio_Brumadinho_impa cto_sa\%C3\%BAde_01_fev_b.pdf

22. Conselho Nacional dos Direitos Humanos Relatório da missão emergencial a Brumadinho/MG após rompimento da Barragem da Vale S/A - Brasília: Conselho Nacional dos Direitos Humanos; 2019. 1를 ed. Disponível em: https://www.mdh.gov.br/todas-as-noticias/2019/fevereiro/missao-emergencial-docndh-apresenta-relatorio-sobre-rompimento-de-barragem-davale/RelatrioMissoemergencialaBrumadinho.pdf

23. Ramos, JO. Prevenção de riscos ambientais e geológicos nos espaços territoriais urbanos da cidade de Teófilo Otoni [Mestrado]. Teófilo Otoni: UFVJM, 2017. 77 pag. [Internet]. [acesso em 2019 jan 10] Disponível em: http://acervo.ufvjm.edu.br/jspui/handle/1/1689

24. Prefeitura Municipal de Teófilo Otoni. Dossiê de tombamento Praça Tiradentes Teófilo Otoni. 2007. [Internet]. [acesso em 2019 jan 10]. Disponível em: http://www.teofilootoni.mg.gov.br/wp-content/uploads/2018/02/Dossi\%C3\%AA-CPPra\%C3\%A7a-Tiradentes-Te\%C3\%B3filo-Otoni.pdf

25. Brasil. Constituição da República Federal. Art. 196. Capítulo II Da Seguridade Social.1988. [Internet]. [acesso em 2019 jan 10]. Disponível em: https://www.senado.leg.br/atividade/const/con1988/con1988_06.06.2017/art_196_.a $\mathrm{sp}$

26. SobralA, FreitasCM, AndradeAV, LyraGFD, MascarenhasMS, AlencarMRF, CastroRAL, França RF. Desastres naturais - sistemas de informação e vigilância: uma revisão da literatura. Epidemiol. Serv. Saúde. 2010; 19(4):389-402. Disponível em: http://scielo.iec.gov.br/pdf/ess/v19n4/v19n4a09.pdf

27. Cerutti DF, Oliveira MLC. Aplicação da gestão de risco de desastres no Sistema Único de Saúde (SUS). Cad. Saúde Colet. 2011; 19 (4): 417-24. Disponível em:http://bvsms.saude.gov.br/bvs/publicacoes/aplicacao_gestao_risco_desastres_su s.pdf

28. Rocha MW. Preparação de profissionais de enfermagem para assistência intrahospitalar em situações de desastres socioambientais [Mestrado]. Rio de Janeiro: Universidade Federal do Rio de Janeiro; 2018. [Internet]. [acesso em 2019 jan 10]. Disponível em: 
http://objdig.ufrj.br/51/teses/871934.pdf?fbclid=IwAR3jwMukHAKP4HmU97shMPQkGDVzWQLGkCG3Ltdtnjj6twfZwt-ysfJB2Y

29. Oliveira WA, Pompeu ELT. O papel da atenção básica nos desastres de origem natural no Brasil. Revista de Saúdeda Faciplac Brasília, 2(1), Dez

2015. [Internet]. [acesso em 2019 jan 10]. Disponível em: file://C:/Users/user/Downloads/103-198-1SM\%20(5).pdf

30. Unasus. Gestão local de desastres naturais para a Atenção Básica. São Paulo. 2016. [Internet]. [acesso em 2019 jan 10]. Disponível em: file://C:/Users/ad/Downloads/_DAB_compilado\%20(1).pdf

31. Silva MA, Carvalho R. Situação de desastre: atuação da equipe de enfermagem em cirurgias emergenciais. Rev. SOBECC. 2013 abr; 18 (2): 67-76. [Internet]. [acesso em 2019 jan 10]. Disponível em:

http://www.sobecc.org.br/arquivos/artigos/2012/pdf/Ano18_n2_\%20abr_jun2013_5.p df

32. Rocha V, Ximenes EF, Carvalho ML, Alpino TMA, Freitas CM. O tema desastre na área da saúde: perfil da produção técnico-científica na base de dados especializada em desastres da Biblioteca Virtual em Saúde (BVS). Ciência \& Saúde Coletiva, 19(9): 3775-3790, 2014. [Internet]. [acesso em 2019 jan 10]. Disponível em: http://www.scielo.br/pdf/csc/v19n9/1413-8123-csc-19-09-3775.pdf

33. Bueno GF, Carvalho R. Assistência de enfermagem no período transoperatório em pacientes vítimas de trauma [monografia]. São Paulo: Faculdade de Enfermagem do Hospital Israelita Albert Einstein; 2009/ 14. Ferreira LR, Carvalho R. Assistência de enfermagem no período transoperatório em pacientes vítimas de trauma: revisão de literatura [monografia]. São Paulo: Faculdade de Enfermagem do Hospital Israelita Albert Einstein; 2009.

34. Conselho Federal de Enfermagem (BR). Resolução COFEN-293/2004. Fixa e estabelece parâmetros para o dimensionamento do quadro de profissionais de enfermagem nas unidades assistenciais das instituições de saúde e assemelhados. 2004 [citado 27 out 2013]. [Internet]. [acesso em 2019 jan 10]. Disponível em: http://www.cofen.gpv.br/resoluocofen-2932004_4329.html.

35. Farias SMC, Teixeira OLC, Moreira W, Oliveira MAF, Pereira MO. Caracterização dos sintomas físicos de estresse na equipe de pronto atendimento. Rev Esc Enferm USP 2011; 45(3):722-9. [Internet]. [acesso em 2019 jan 10]. Disponível em: http://www.scielo.br/pdf/reeusp/v45n3/v45n3a25.pdf

36. Oliveira WA. Atuação da enfermagem em emergências de saúde pública no brasil. [Internet]. [acesso em 2019 jan 10]. Disponível em: http://revista.faciplac.edu.br/index.php/REFACl/article/view/196/83

37. Luchtemberg MN, Pires DEP. Enfermeiros do Serviço de Atendimento Móvel de Urgência: perfil e atividades desenvolvidas. Rev Bras Enferm [Internet]. 2016 marabr;69(2):213-20. [acesso em 2019 jan 10]. Disponível em: http://www.scielo.br/pdf/reben/v69n2/0034-7167-reben-69-02-0213.pdf 
38. Brasil. Secretaria de Estado da Saúde Governo do Estado de São Paulo. Desastres e Incidentes com Múltiplas Vítimas Plano de Atendimento - Preparação Hospitalar. 2012. [Internet]. [acesso em 2019 jan 10] Disponível em: http://www.saude.sp.gov.br/resources/ses/perfil/gestor/homepage/desastres/prepara cao-hospitalar-para-atendimento-de-desastres-e-incidentes-com-multiplasvitimas/incidentes_com_multiplas_vitimas_e_desastres_2012.pdf

39. Fernandes VC. Acidente com múltiplas vítimas: Uma análise do planejamento e preparação do cuidado de enfermagem na sala de emergência. [Mestrado]. Universidade Federal do Rio de Janeiro (UFRJ) Rio de Janeiro. 2010. [Internet]. [acesso em 2019 jan 10]. Disponível em:

http://objdig.ufrj.br/51/dissert/EEAN_M_VladimirChavesFernandes.pdf

40. Marousky RT. Planejamento de desastres: idéias realistas para a sala de cirurgia. AORN J. 1992;56(4):679-87.

41. Brasil. Ministério da Saúde. Portaria n 2.436, de 21 de setembro de 2017. Dispõe sobre a prova a Política Nacional de Atenção Básica, estabelecendo a revisão de diretrizes para a organização da Atenção Básica, no âmbito do Sistema Único de Saúde (SUS). [Internet]. [acesso em 2019 jan 10]. Disponível em: http://bvsms.saude.gov.br/bvs/saudelegis/gm/2017/prt2436_22_09_2017.html

42. Ferreira SRS, Périco LAD, Dias VRFG. A complexidade do trabalho do enfermeiro na Atenção Primária à Saúde. Rev Bras Enferm [Internet]. 2018;71(supl1):752-7. [acesso em 2019 jan 10]. Disponível em:

http://www.scielo.br/pdf/reben/v71s1/pt_0034-7167-reben-71-s1-0704.pdf

43. Organização Pan-Americana da Saúde. Desastres naturais: proteger a saúde do público. Washington, DC: PAHO; 2000. Scientific Publication No. 575. [Internet]. [acesso em 2019 jan 10]. Disponível em:

http://www.paho.org/hq/dmdocuments/2010/9275115753.pdf

44. Roth $P$, Simanello MA. Promoção da saúde da família durante as transições. In: Bomar PJ. Promovendo a saúde nas famílias. Aplicando Pesquisa e Teoria Familiar à Prática de Enfermagem. 3.ed New York: Elsevier; 2004. p. 477-506.

45. Brasil. Ministério da Saúde Secretaria de Vigilância em Saúde plano nacional de preparação e resposta às emergências de saúde pública Guia de Preparação e Resposta aos Desastres Associados às Inundações para a Gestão Municipal do Sistema Único de Saúde Julho/2011. Disponível em: http://portalarquivos.saude.gov.br/images/pdf/2017/junho/08/Guia_para_sms_desast res_julho_2011.pdf 